\title{
Critical exponents of the disorder-driven superfluid-insulator transition in one-dimensional Bose-Einstein condensates
}

\author{
J. C. C. Cestari, ${ }^{1}$ A. Foerster, ${ }^{1}$ M. A. Gusmão, ${ }^{1}$ and M. Continentino ${ }^{2}$ \\ ${ }^{1}$ Instituto de Física, Universidade Federal do Rio Grande do Sul, Porto Alegre, Rio Grande do Sul, Brazil \\ ${ }^{2}$ Centro Brasileiro de Pesquisas Físicas, Rio de Janeiro, Brazil
}

(Received 14 June 2011; published 7 November 2011)

\begin{abstract}
We investigate the nature of the superfluid-insulator quantum phase transition driven by disorder for noninteracting ultracold atoms on one-dimensional lattices. We consider two different cases: Anderson-type disorder, with local energies randomly distributed, and pseudodisorder due to a potential incommensurate with the lattice, which is usually called the Aubry-André model. A scaling analysis of numerical data for the superfluid fraction for different lattice sizes allows us to determine quantum critical exponents characterizing the disorder-driven superfluid-insulator transition. We also briefly discuss the effect of interactions close to the noninteracting quantum critical point of the Aubry-André model.
\end{abstract}

DOI: 10.1103/PhysRevA.84.055601

PACS number(s): 67.85.Hj, 64.60.an, 64.70.Tg

Introduction. A superfluid-insulator transition in a disordered noninteracting system of bosons at zero temperature is a special type of quantum phase transition (QPT) [1]. Instead of the more conventional competition between different interactions, it is disorder that causes a drastic change in the nature of the ground state, thus altering the physical characteristics of the material. A similar type of transition from a metal to an insulator, usually called the Anderson localization transition, was first proposed by Anderson [2] and has been extensively studied in electronic systems [3]. In general, the approach focuses on the conductance behavior as the Fermi level changes in the vicinity of the mobility edge, which separates localized and extended one-particle states. In this context, the lower critical dimension has been determined to be $d_{L}=2$, which means that all the states are localized in one dimension for any finite amount of disorder. Nevertheless, given that the states in a strictly nondisordered system are extended, there is a clear change of regime when the disordered strength is reduced to zero, which can be characterized as a QPT.

In the past decade, enormous progress in the techniques for creating ultracold atom systems in laboratory settings extended the interest in the disorder effects and localization to bosonic systems (for recent reviews, see [4-7]). For bosons, the transition is from the insulator (localized) state to the superfluid one. It was observed both for laser-speckle disorder [8] and quasiperiodic optical lattices [9] in Bose-Einstein condensates of ${ }^{87} \mathrm{Rb}$ and ${ }^{39} \mathrm{~K}$ atoms, respectively. While speckle disorder comes close to the Anderson-type random disorder considered in theoretical approaches, quasiperiodic lattices present a superposition of the lattice potential with an incommensurate one and can be viewed as experimental realizations of pseudodisorder models like the Aubry-André (AA) model [10]. The latter also shows superfluid and localized regimes in one dimension, but the transition between them occurs at a nonzero critical disorder [10,11].

Recently, we have investigated numerically the superfluidinsulator transition in one-dimensional, noninteracting systems of bosons with these two types of disorder [12]. Here we focus on the scaling properties of the superfluid fraction near the superfluid-insulator transition, obtaining the relevant critical exponents. For random disorder, even though the superfluid phase is destroyed for arbitrarily weak disorder, we show that the transition can still be described as a quantum critical phenomenon with well-defined critical exponents and power-law scaling behavior. The same happens for the AA model, but the universality classes are different.

Our starting point is a well-known scaling relation for the singular part of the superfluid density $\rho_{s}$ close to a quantum superfluid-insulator phase transition [13],

$$
\rho_{s} \sim|g|^{\nu(d+z-2)}
$$

where $g$ measures the distance to the quantum critical point (QCP), $v$ is the correlation length exponent (i.e., the correlation length diverges as $\xi \sim|g|^{-\nu}$ at the QCP), $d$ is the spatial dimension, and $z$ is the dynamic critical exponent associated with the QCP. The superfluid density is directly related to the helicity modulus [14] and can be viewed as a measure of the system's response to a phase-twisting field. Thus, it is natural to interpret the correlation length as a phase-coherence length. In the insulating phase, it should coincide with the localization length, which measures the spatial extent of the wave functions. This holds also for disordered metals [3].

In a finite system, even at criticality, the correlation length is limited by the system size $L$, and the finite-size-scaling form of the superfluid density is

$$
\rho_{s} \sim L^{-(d+z-2)} F(L / \xi)=L^{-(d+z-2)} F\left(L|g|^{\nu}\right) .
$$

The corresponding relation for the superfluid fraction $\left(f_{s}=\right.$ $\left.L^{d} \rho_{s}\right)$ is

$$
f_{s} \sim L^{-(z-2)} F\left(L|g|^{\nu}\right) .
$$

This last equation is suitable to determine the critical exponents $v$ and $z$ from a numerical evaluation of $f_{s}$ for various lattice sizes, as we do in the following.

Anderson-like disorder. The usual Hamiltonian describing interacting bosons on a lattice is known as the Bose-Hubbard Hamiltonian and is given by

$$
H=\sum_{i} \varepsilon_{i} n_{i}+\Omega \sum_{\langle i j\rangle}\left(a_{i}^{\dagger} a_{j}+a_{j}^{\dagger} a_{i}\right)+\frac{U}{2} \sum_{i} n_{i}\left(n_{i}-1\right),
$$


where $a_{i}^{\dagger}$ and $a_{i}$ are the creation and annihilation operators of a boson at the lattice site $i, n_{i}=a_{i}^{\dagger} a_{i}$ is the corresponding number operator, each site has a single bound state of energy $\varepsilon_{i}$, hopping between sites is restricted to nearest neighbors, with amplitude $\Omega$, and $U$ is a local repulsive interaction. In the rest of this paper, energies are measured in units of the tunneling amplitude $\Omega$. An Anderson-like disorder [2] is introduced by choosing random local energies with a uniform distribution in the range $-\Delta / 2 \leqslant \varepsilon_{i} \leqslant \Delta / 2$, so that $\Delta$ is a measure of the disorder strength.

We carried out a thorough numerical analysis of the above model for the noninteracting case in one spatial dimension. Details of this numerical study are given in Ref. [12]. We recall one of the main results reported there, namely that the superfluid fraction for a lattice of size $L$ obeys the relation $f_{s}=\exp \left(-\Delta / \Delta_{L}\right)^{4 / 3}$, where $\Delta_{L}$ is a characteristic disorder strength for suppression of superfluidity, which scales with the lattice size as $\Delta_{L}=C L^{-3 / 2}$. This latter relation is consistent with the expected value of the critical disorder strength $\Delta_{c}=0$ for destroying the superfluid phase in the thermodynamic limit for a one-dimensional system. Furthermore, defining $g \equiv \Delta-$ $\Delta_{c}=\Delta$, this scaling of $\Delta_{L}$ with $L$ is recognized as the finitesize version of the general relation $\xi \sim|g|^{-v}$, immediately yielding the correlation-length exponent $v=2 / 3$.

Equation (3) implies that $L^{z-2} f_{s}$ is a universal function of $L \Delta^{\nu}$. The corresponding plot of our data for different lattice sizes is shown in Fig. 1, where it is clear that all the data collapse onto a universal curve. The appearance of $f_{s}$ alone as the scaling quantity in the vertical axis means that the dynamic critical exponent is $z=2$. The scaled variable of the horizontal axis in the collapsed plot confirms the value $v=2 / 3$ for the correlation-length exponent. Actually, for the present problem, we are able to determine explicitly the scaling function $F(x)$ in Eq. (3). The above-mentioned expression for $f_{s}$ implies that $F(x)=\exp \left(-x^{2} / C^{2}\right)$. Since $x=L / \xi=L \Delta^{v}$, the value $z=2$ for the dynamic exponent implies a jump of the superfluid fraction from 0 to 1 at the transition occurring for

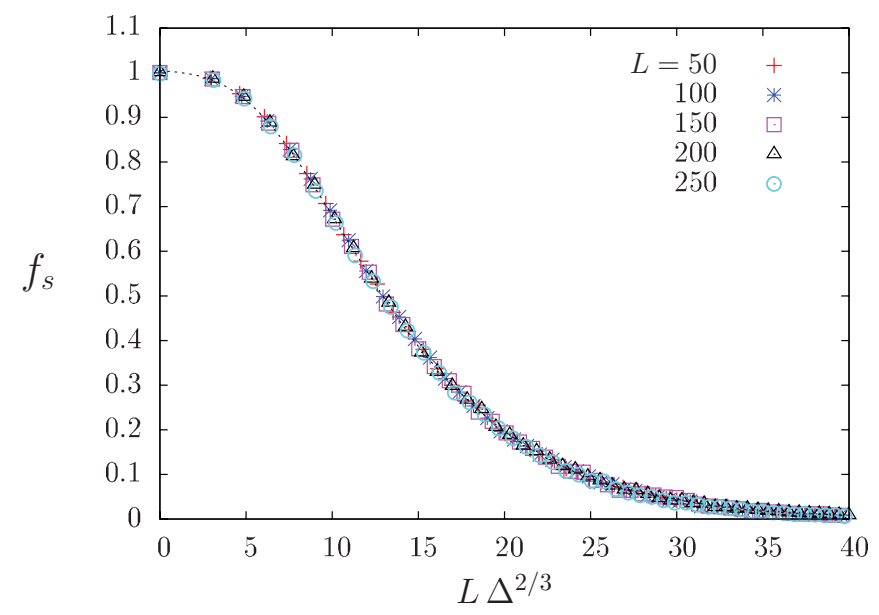

FIG. 1. (Color online) Finite-size scaling of the superfluid fraction for Anderson-type disorder. According to Eq. (3), the horizontalaxis variable for collapsed curves is $L|g|^{v}$, which gives $v=2 / 3$, while the absence of any rescaling of $f_{s}$ implies that $z=2$.
$\Delta=0$ in the limit $L \rightarrow \infty$. This jump is reminiscent of that of the helicity modulus in the two-dimensional $X Y$ model [15].

The value $v=2 / 3$ obtained here for the correlation-length exponent has not been determined previously, to the best of our knowledge. This new exponent for the superfluid-insulator transition seems to violate the inequality $v \geqslant 2 / d$, which holds for other disordered systems [13]. However, this inequality has been proved only for interacting systems and for nonzero critical values of the parameter driving the transition, which is not the case here. On the other hand, the dynamic exponent $z=$ 2 implies that the effective dimension of the quantum phase transition [1] is $d_{\mathrm{eff}}=d+z=3$. For disordered interacting bosons, the Bose-glass-to-superfluid transition is characterized by the relation $z=d$ [13]. Therefore, interacting and noninteracting bosons are in different universality classes with respect to the localization transition. In the renormalization group language, interaction is a relevant term close to the disordered noninteracting fixed point.

Aubry-André model. The Aubry-André model can also be described by the Hamiltonian (4), with $U=0$, except that the distribution of local energies is not random but periodic with a period incommensurate with the lattice spacing. These energies are usually written as

$$
\varepsilon_{i}=\Delta \cos (2 \pi \beta i)
$$

where $\beta=(1+\sqrt{5}) / 2$ is the golden ratio and $i$ assumes integer values from 1 to $L$. This is actually a special case of the Harper model [16] for electrons in a two-dimensional lattice in the presence of a perpendicular magnetic field, for which Eq. (5) holds for any value of $\beta$, with different characteristics of the spectrum for rational or irrational values. Disorder-like effects here are a consequence of the incommensurability between the "external potential" and the lattice. Aubry and André [10] proved that for this model localization occurs only when the strength of the potential $\Delta$ is larger than the critical value $\Delta_{c}=2$. For finite lattices, it is convenient to replace $\beta$ with $\beta_{n}=F_{n+1} / F_{n}$, the ratio of two consecutive Fibonacci numbers, whose limit for $n \rightarrow \infty$ is the golden ratio. Then, the lattice size must be chosen as $L=F_{n}$ in order to allow for the use of periodic boundary conditions. For this kind of finite lattices, the critical value $\Delta_{c}=2$ remains a rigorous result [11], since it corresponds to a duality between the Hamiltonians in position and momentum space.

It was shown in Ref. [12] that the superfluid fraction undergoes a very sharp transition around $\Delta=2$ for essentially all lattice sizes. This sharpness makes it difficult to directly extract the correlation-length exponent, as done for random disorder. Here, we concentrate on a narrow region around $\Delta_{c}$, searching for the appropriate scaling variable proportional to $g \equiv \Delta-\Delta_{c}=\Delta-2$, and the appropriate scaling of the superfluid fraction. Our results are shown in Fig. 2. The data collapse onto two universal curves, for even and odd numbers of lattice sites. Although the scaling functions are different for these two cases, the critical exponents for which the curves collapse are the same. In view of Eq. (3), we immediately identify the correlation-length exponent $v=1$ from the $x$-axis scaling variable in Fig. 2 and the dynamic exponent $z=2.374$ from the $y$-axis scaling. It was already known [10] that $v=1$ 


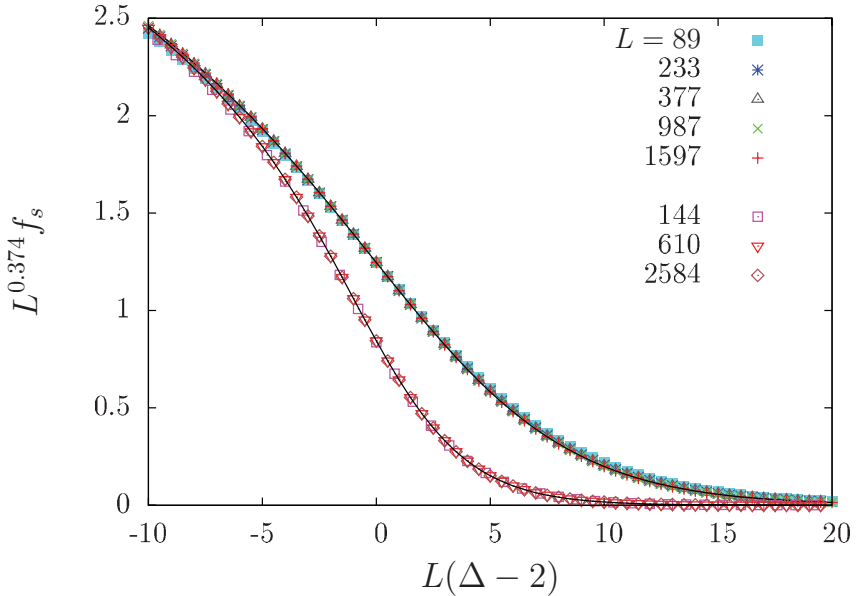

FIG. 2. (Color online) Finite-size scaling of the superfluid fraction for the AA model. The data collapse in different curves for even and odd numbers of lattice sites (respectively, lower and upper curves).

for this model. Next we discuss the obtained value of $z$ in the light of properties of the energy spectrum.

The spectrum of the Harper model has been thoroughly studied in the past [17-21]. For general rational values of $\beta$ it is multifractal at $\Delta=2$, yielding the famous Hofstadter butterfly [22], shown in Fig. 3. There we highlight the case that we are studying here, with $\beta$ being a rational approximant of the golden ratio. In particular, the figure shows our result obtained from numerical diagonalization of the Hamiltonian for a lattice of size $L=610$. The two bottom panels illustrate the fractal nature of this spectrum.

With our replacement of $\beta$ by a ratio of two Fibonacci numbers, $\beta_{n}=F_{n+1} / F_{n}$, the spectrum is equivalent to the one for $\bar{\beta}_{n}=\beta_{n}-1=F_{n-1} / F_{n}$, which contains $F_{n}$ bands and $F_{n-1}$ gaps. As discussed in detail in Refs. [19,20], when $F_{n}=L \rightarrow \infty$ the width $\Delta E_{L}$ of a given band belonging to the spectrum scales as $\Delta E_{L} \sim L^{-\gamma}$, with different regions of the spectrum associated with different values of $\gamma$ (not to be confused with the susceptibility critical exponent). In particular, a maximum value $\gamma_{\max }=2.374$ corresponds to band-edge states. On the other hand, the band width is a characteristic energy of the system and therefore should scale as $\xi^{-z}$, which means that $\Delta E_{L} \sim L^{-z}$. Our finding of $z=\gamma_{\max }$ is in agreement with the relevant state for the zero-temperature superfluid-insulator transition being the bottom edge of the lowest-lying band.

Effects of interaction. The interaction term in Eq. (4) can be treated as a relevant field close to the QCP. The knowledge of the dynamic exponent allows us to generalize the scaling relations close to the QCP for small but finite $U$. The free energy, for example, is given by

$$
F_{s} \propto|g|^{\nu(d+z)} P\left(U /|g|^{\nu z}\right),
$$

where again we used the fact that $U$ is an energy and thus scales with $\xi^{-z}$. From the above equation, we see that the scaling contribution to the critical line separating the superfluid and insulating phases is

$$
U_{c}(g) \propto|g|^{v z} .
$$

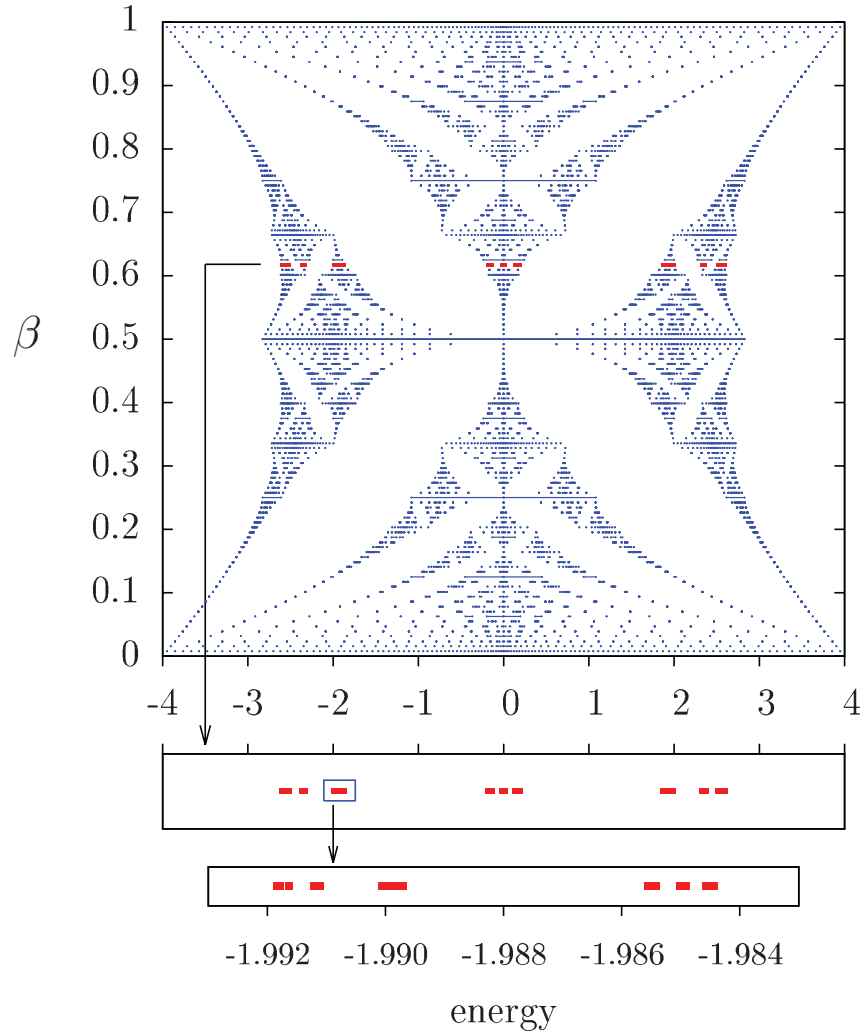

FIG. 3. (Color online) Spectra of the Harper model, highlighting the spectrum corresponding to the AA model (for a rational approximation of the golden ratio $\beta=987 / 610$ ). Its fractal nature is illustrated in the bottom by expanding the small box drawn inside the middle panel. We show the spectrum for $\beta-1$, which is the same as for $\beta$ according to Eq. (5).

The critical exponents are those associated with the disordered noninteracting QCP at $U=0, \Delta=\Delta_{C}=2$. Since the product $v z=2.374$ is large we have to take into account analytic contributions to the shape of the critical line. This line can be written in general as

$$
U_{c}=f(g)+a_{ \pm}|g|^{\nu z},
$$

where $f(g)$ is an analytic function and \pm refers to the sign of $g$. Expanding close to the QCP, analytic contributions up to the second order dominate over the scaling term when $g \rightarrow 0$. To illustrate this point, in Fig. 4 we show a phase diagram close to the noninteracting fixed point at $\Delta_{c}=2$, for repulsive $(U>0)$ and attractive $(U<0)$ interactions. This is a plot of the superfluid fraction (color scale) as a function of $\Delta$ and $U$, obtained by diagonalizing the Hamiltonian (4) for $N=8$ interacting bosons on a lattice of $L=8$ sites. We can see that a straight line [i.e., $f(g) \sim g$ ] is a very good approximation to the boundary between the superfluid and localized regions. Even though the transition is smoothed out by the small lattice size, it is worth mentioning that the value of $f_{s}$ at the critical point $(U=0, \Delta=2)$ is compatible with the lower curve of Fig. 2 for $L=8$.

Conclusions. We studied the superfluid-insulator transition for bosons on a one-dimensional lattice, both with random disorder and the pseudodisorder described by the Aubry-André model, the two prototype models employed in the investigation 


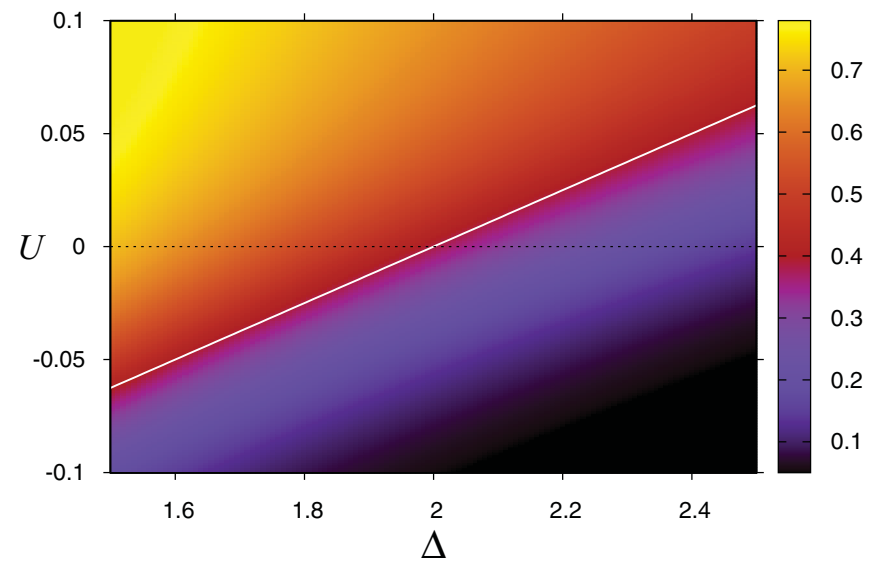

FIG. 4. (Color online) Zero-temperature phase diagram near the localization transition in the presence of a small interaction. The color scale indicates values of the superfluid fraction. The line $U_{c}(g)$ in the vicinity of the non-interacting QCP is essentially linear (with a slope close to 0.1$)$, implying that it is dominated by the analytic part $f(g)$ in Eq. (8).

of localization for ultracold atoms in optical lattices. Using a finite-size-scaling analysis of the superfluid fraction, we obtained the critical exponents characterizing this transition. The superfluid fraction yields the correlation-length exponent $v$ and the dynamic critical exponent $z$. For random disorder we found $v=2 / 3$ and $z=2$, while for the AA model the results are $v=1$ and $z=2.374$. The other critical exponents can be obtained from the quantum hyperscaling relations [1] $2-\alpha=$ $v(d+z)$ and $2 \beta=v(d+z-2+\eta)$. These two models fall into different universality classes, which is not surprising since the critical disorder strength for the superfluid-insulator transition is zero for Anderson-like disorder and nonzero for the AA model, which also exhibits a multifractal energy spectrum at the QCP.

It is interesting to observe that the scaling form of the free energy for nonzero temperature $T$ can be used to determine the thermodynamic behavior close to the superfluid-insulating QCP. From it, a general dependence of the specific heat with temperature is obtained [13], with the form $C \sim T^{d / z}$.

Our brief discussion of interaction effects in the AA model shows that the critical point moves to stronger disorder for repulsive interaction and to weaker disorder in the attractive case. Regions of the phase diagram that correspond to localized and superfluid regimes in the thermodynamic limit are separated by a line that is approximately linear, reflecting the dominance of nonsingular contributions.

Acknowledgments. This work was supported in part by CNPq, Conselho Nacional de Desenvolvimento Científico e Tecnológico (Brazil). M.A.C. also thanks FAPERJ, Fundação de Amparo à Pesquisa do Estado do Rio de Janeiro, for partial financial support.
[1] M. A. Continentino, Quantum Scaling in Many-Body Systems (World Scientific, Singapore, 2001).

[2] P. W. Anderson, Phys. Rev. 109, 1492 (1958).

[3] P. A. Lee and T. V. Ramakrishnan, Rev. Mod. Phys. 57, 287 (1985).

[4] L. Fallani, C. Fort, and M. Inguscio, in Advances in Atomic, Molecular, and Optical Physics, edited by E. Arimondo, Vol. 56 (Academic Press, San Diego, CA, USA, 2008), pp. 119-160.

[5] A. Lagendijk, B. van Tiggelen, and D. S. Wiersma, Phys. Today 62, 24 (2009).

[6] A. Aspect and M. Inguscio, Phys. Today 62, 30 (2009).

[7] L. Sanchez-Palencia and M. Lewenstein, Nat. Phys. 6, 87 (2010).

[8] J. Billy, V. Josse, Z. Zuo, A. Bernard, B. Hambrecht, P. Lugan, D. Clément, L. Sanchez-Palencia, P. Bouyer, and A. Aspect, Nature (London) 453, 891 (2008).

[9] G. Roati, C. D'Errico, L. Fallani, M. Fattori, C. Fort, M. Zaccanti, G. Modugno, M. Modugno, and M. Inguscio, Nature (London) 453, 895 (2008).
[10] S. Aubry and G. André, Ann. Israel Phys. Soc. 3, 133 (1980).

[11] G.-L. Ingold, A. Wobst, C. Aulbach, and P. Hanggi, Eur. Phys. J. B 30, 175 (2002).

[12] J. C. C. Cestari, A. Foerster, and M. A. Gusmão, Phys. Rev. A 82, 063634 (2010).

[13] M. P. A. Fisher, P. B. Weichman, G. Grinstein, and D. S. Fisher, Phys. Rev. B 40, 546 (1989).

[14] M. E. Fisher, M. N. Barber, and D. Jasnow, Phys. Rev. A 8, 1111 (1973)

[15] K. Harada and N. Kawashima, Phys. Rev. B 55, R11949 (1997).

[16] P. G. Harper, Proc. Phys. Soc. London, Sect. A 68, 874 (1955).

[17] M. Kohmoto, Phys. Rev. Lett. 51, 1198 (1983).

[18] J. B. Sokoloff, Phys. Rep. 126, 189 (1985).

[19] C. Tang and M. Kohmoto, Phys. Rev. B 34, 2041 (1986).

[20] H. Hiramoto and M. Kohmoto, Phys. Rev. B 40, 8225 (1989).

[21] H. Hiramoto and M. Kohmoto, Int. J. Mod. Phys. B 6, 281 (1992)

[22] D. R. Hofstadter, Phys. Rev. B 14, 2239 (1976). 